\title{
Impact of High-Cost Drugs for Individual Patient Use
}

\author{
Gisselle Gallego, Terry Melocco, Susan J Taylor, Jo-anne E Brien
}

\begin{abstract}
Aim: To document and describe the individual patient use (IPU) scheme at St Vincent's Hospital, Sydney in terns of submissions and approvals and assess the financial impact of the scheme on the hospital drug expenditure.

Method: All submissions for IPU approvals received between January 1997 and December 2001 were reviewed. Submissions were collected on a calendar year basis. Data collection and analysis included identification of approved medication and indication, off-label or approved indication, prescriber, ward, outcome of therapy, person deciding the approval, approval date duration and expiry, amount of medication dispensed and the cost of therapy. The annual cost and proportion of overall drug expenditure for each approval was calculated.

Results: The number of approvals had a trend to increase each year. $67.1 \%$ of the IPU approvals were for off-label indications. Requested feedback on clinical outcomes was provided only in $18 \%$ of cases. The drug acquisition cost of the IPU scheme more than doubled in the period between 1999 and 2001. Similarly the proportion of the drug expenditure on IPU drugs increased significantly $(\mathrm{p}<0.001)$ from $1.6 \%$ in 1999 to $3.6 \%$ in 2001.

Conclusion: The results indicated that the number of approvals and submissions for IPU had a tendency to increase. The financial impact of the IPU scheme increased over the years reviewed.
\end{abstract} J Pharm Pract Res 2004; 34: 100-3.

\section{INTRODUCTION}

There are different mechanisms to fund medications in the Australian healthcare system. One of these mechanisms is the Pharmaceutical Benefits Scheme (PBS), which subsidises drugs dispensed in community pharmacies and private hospitals and is funded by the Commonwealth Government. 'A number of public hospitals in some States have implemented the PBS as a funding mechanism for outpatient and discharge medications. ${ }^{2}$

The Commonwealth Government also provides funding to the States and Territories for Section 100 or the Highly Specialised Drugs (HSD) Program. These are expensive drugs used for chronic conditions such as cancer, HIV and organ transplantation. They are prescribed by specialists and dispensed through selected hospital pharmacies. Hospitals are responsible for the drug cost of inpatients on HSD. If drugs are prescribed for approved conditions, reimbursement is sought by the hospital, otherwise the hospital will incur the whole cost.'

The Commonwealth Government gives funding to the States and Territories for public hospitals and other

Gisselle Gallego, BPharm Chem, PhD candidate, Faculty of Pharmacy, The University of Sydney, Camperdown, Terry Melocco, BPharm, Director of Pharmacy, St Vinceni's Hospital, Darlinghurst, Susan J Taylor, BPharm, MSc (Clinical), PhD, Senior Lecturer, Jo-anne E Brien, BPhann, BS (Pharm), PharmD, Pharmacia Chair in Clinical Pharmacy (St Vinceni's Hospital). Faculty of Pharmacy. The University of Sydney, Camperdown. New South Wales

Address for correspendence: Professor Jo-aunc Brien, Faculty of Pharmacy, The University of Sydney, Building A 15, Camperdown NSW 2006, Australia E-mail: jebricn@pharm.usyd.edu.au state-based programs. ${ }^{3}$ In contrast to the uncapped budget for the PBS and HSD program, public hospitals deal with capped budgets and scarce resources. ${ }^{34}$ In order to deal with these restrictions, public hospitals need to have cost containment strategies in place. 5.6 The Drug and Therapeutics Committee (D'TC) at St Vincent's Hospital, Sydney has the task of constraining drug costs and ensuring the quality use of medicines. By analysing drug usage trends the DTC has become more involved in cost containment and predicting budgetary needs.

The individual patient use (IPU) scheme was created as a gatekeeping device for high-cost drugs. IPU drugs are often requested for rare diseases where the drug has limited evidence as standard treatment and for nonapproved indications. No funding from the States, Territories or the Commonwealth Government is provided for these drugs. They are generally high-cost drugs used for a specific therapeutic indication. These drugs are not available on the hospital formulary, nor have they been approved by the hospital for the intended indication. Therefore, the JPU scheme is used to allow prescribers to access non-formulary drugs in exceptional cases.

The IPU scheme is managed through the DTC, which faces certain difficulties. One of these difficulties is the limited information available to conduct a complete evaluation of safety and efficacy for these drugs.

These drugs can also represent a new therapeutic modality such as biological therapy. Evidence supporting applications through the IPU scheme may come from case reports or case series of a small number of patients with a single exposure. Estimates of cost effectiveness or any other economic evaluation may be missing or poorly documented due to the fact that, in the majority of cases, the indication is not a Therapeutic Goods Administration (TGA) approved indication (off-label).

All of these facts bring a range of economic, legal and ethical issues to the IPU scheme. Even though hospitals may have developed guidelines for use and approval of high-cost drugs as a cost-containment strategy, it is difficult to readily assess the outcomes of the IPU scheme. Outcomes of interest include the clinical consequences for the individual patient, as well as the healthcare costs involved. Comprehensive data are difficult to collect and interpret since there are limitations in documentation, follow-up, and availability of comparative and financial data. Consequently, the purpose of this study was to docunent and describe the IPU scheme at St Vincent's Hospital, Sydney and assess the financial impact on the hospital drug expenditure. Such review is a basis for evaluation of current processes and consideration of alternative approaches.

\section{METHOD}

The study was conducted at St Vincent's Hospital, Sydney, a 350-bed university-affiliated, tertiary acute care hospital. The IPU scheme was reviewed for consistency of process and use of precedents. All submissions for IPU 
approval received between January 1997 and December 2001 were reviewed. Submissions were collected on a calendar-year basis (January to December). Sources of data included pharmacy department records, drug use evaluation data records, DTC records, and dispensing systems MEDRECORD (version 5.36 DOS) for drugs dispensed before November 1999 and STOCCA (version 3.7) for drugs dispensed after November 1999.

Acquisition prices of drugs were obtained from the 2001 inventory catalogue provided by STOCCA. Information regarding the status of the indication being approved was obtained from different sources including the Schedule of Phannaceutical Benefits, MIMS and the TGA 1800 contact line.$^{7.9}$ Data collection included medication, approved indication, status of the indication being approved, prescriber, ward, outcome of therapy, person deciding the approval, approval date duration and expiry, and the cost of therapy. The annual cost and proportion of overall drug expenditure for each approval was calculated. IPU approvals were excluded if data such as course of therapy and duration were not available and if cost did not exceed $\$ 100$ per year.

\section{Statistical Analysis}

A Microsoft Excel 2000 database was designed to store and analyse data. Data were entered and validated by performing manual checks against original sources. Data for submissions and approvals were grouped per calendar year. Each year was analysed separately to account for alterations in the status of medications and indications that occurred over time. Categories were used to differentially describe available evidence rather than funding source.

- Special access scheme (SAS) unregistered drugs made available under the provision of sections 19 and 18 of the Therapeutic Goods Act. ${ }^{10}$

- Non-TGA approved indication drugs marketed in Australia but not for the intended indication (off-label).

- TGA approved indication and not listed in PBS subsidy criteria.

- TGA approved indication and listed in PBS subsidy criteria.
Data were summarised and the proportion of drug expenditure and annual cost were compared using the chi-square test for linear trend. Significance was set at the $5 \%$ level.

\section{RESULTS}

The process of approval at St Vincent's Hospital is outlined in Figure 1. An IPU request form is filled out by the prescriber and sent to the Director of Pharmacy who, according to the complexity and priority of the request, either approved the request or asked for further evidence to support the indication. Following this, the Director of Pharmacy approved the request or forwarded it to the Chairman of the DTC for a direct decision or referral to the next DTC meeting. At this point, the request was either approved or rejected. However, there were circumstances where acute conditions needed immediate attention and decisions could be made outside the DTC meeting schedule. Once the drugs were approved an annotation was entered in the STOCCA dispensing system, with the date of approval, indication and duration of the therapy.

There were no explicit criteria for IPU approvals. Dose, duration of therapy, indication, failure to previous treatment, rationale for use, evidence for use in the specific indication, available level of evidence, safety, clinical need and cost of course were the minimum requirements for $\mathrm{IPU}$ submissions.

A total of 340 new submissions and 323 approvals for IPU between 1997 and 2001 were reviewed. The process was consistent for the drugs reviewed. Once a drug was approved for a certain indication, submissions for other patients with the same indication were approved. A total of 33 literature reviews were supplied ( $8.8 \%$ of the total submissions).

There was a trend that IPU submissions and approvals had increased through the years (Figure 2). There were a number of medications that despite being approved were never dispensed to the patient (13). Two submissions were withdrawn after being submitted.

IPU approvals for 1997 and 1998 were excluded from further analysis since data such as course of therapy and duration were not available due to limitations in data retrieval from MEDRECORD.
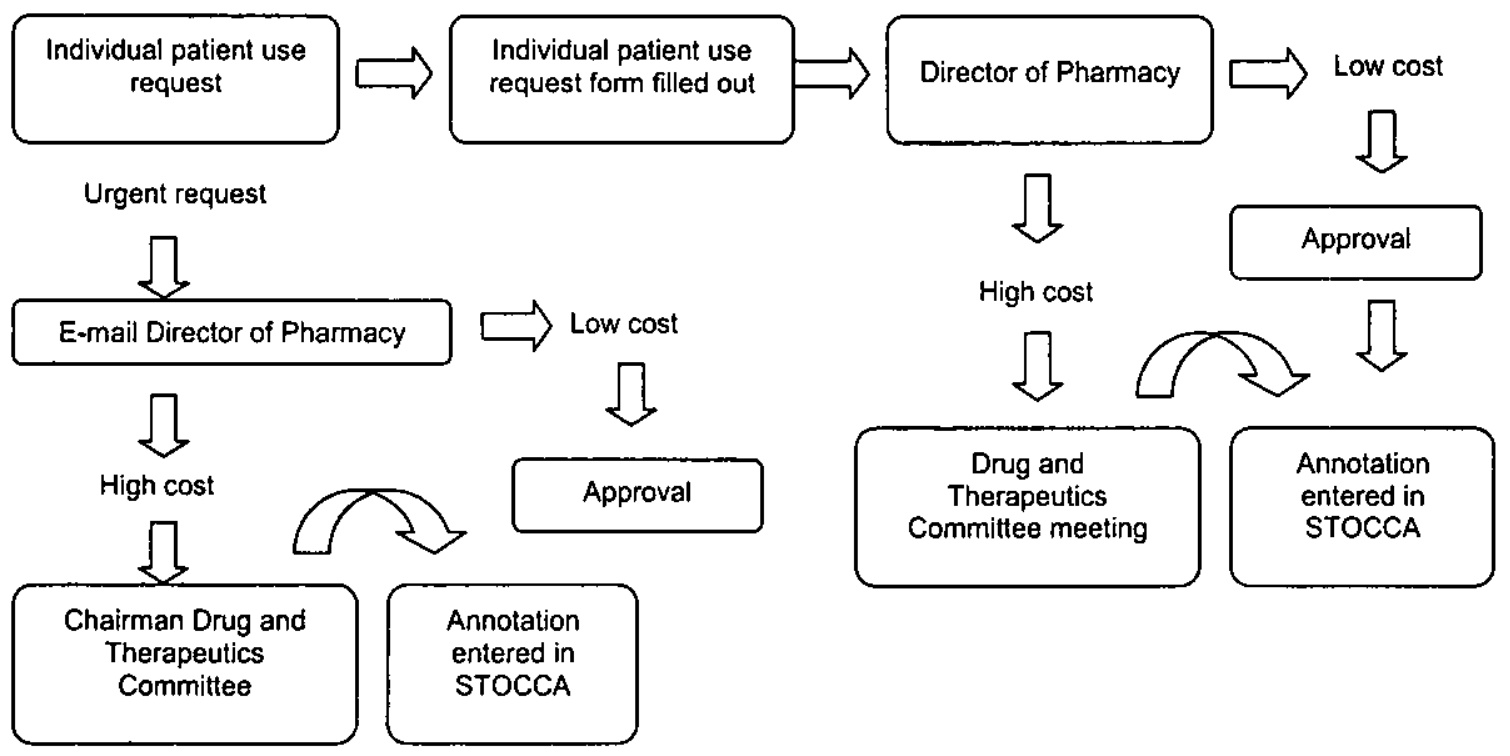

Figure 1. Process of submission and approval of the individual patient use scheme at St Vincent's Hospital 


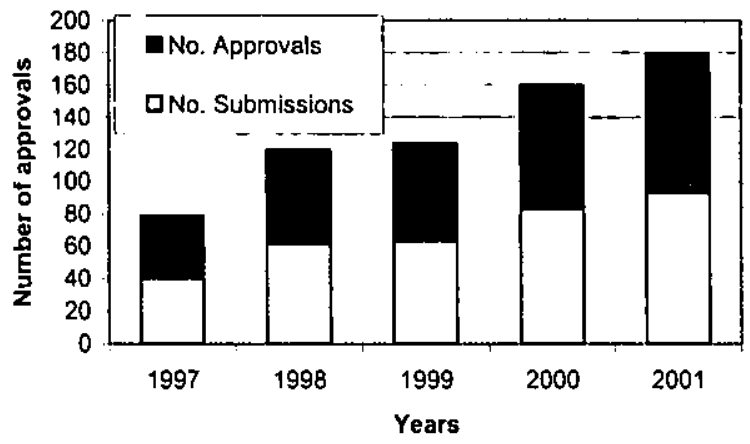

Figure 2. Submissions and approvals for new individual patient use drugs

From 63 submissions in 1999,61 new IPU applications were approved for 31 different medications and 51 patients. Mycophenolate (11 approvals) accounted for $18 \%$ of the approvals, followed by octreotide, erythropoietin and cyclosporin ( $8.2 \%$ each). In 2000, the number of new IPU approvals increased to 77, for 37 medications and 75 patients. Mycophenolate was still the most widely approved $(22.9 \%)$ of the approvals followed by pamidronate (12\%), cyclosporin and tacrolimus (4.8\% each). By 2001, the number of approvals increased to 87 for 20 medications in 79 patients. The medication most frequently approved was pamidronate $(18.4 \%)$ followed by mycophenolate $(17.2 \%)$ and gabapentin $(10.3 \%)$. The approved medications in order of frequency between 1999 and 2001 is listed in Table 1.

Table 1. Most frequently approved individual patient use drugs in order of frequency (1999-2001)

\begin{tabular}{lll}
\hline 1999 & 2000 & 2001 \\
\hline mycophenolate & mycophenolate & pamidronate \\
octrcotide & pamidronate & mycophenolate \\
erythropoietin & cyclosporin & gabapentin \\
cyclosporin & tacrolimus & octreotide \\
pamidronate & octreotide & imatinib \\
interferon alfa 2a & interferon alfa 2a & tacrolimus \\
tacrolimus & domase alfa & linezolid \\
paclitaxel & basiliximab & cnoxaparin \\
& valaciclovir & cyclosporin \\
& & basiliximab \\
\hline
\end{tabular}

More than half of the medications $(67.1 \%)$ were approved for off-label indications (Table 2). This group was followed by medications with indications listed on the PBS but not on the hospital formulary (12.4\%). Third group was those with TGA approval but not funded through the HSD program or PBS (12\%). The last group was the special access scheme (SAS) medications with $6.7 \%$. The 'other' classification in 2000 represents a single approval of a tensoactive agent.

A report of clinical outcomes was requested for 71 out of the 225 approvals $(31.5 \%)$. Of these 13 out of 71 (18\%) were reported. The percentage of outcomes requested was exactly the same in 1999 and 2001. During
Table 2. Status of indications of drugs approved expressed as percentages of the total number of individual patient use approvals

\begin{tabular}{lcccc}
\hline Stutus & $\begin{array}{c}\mathbf{1 9 9 9} \\
(\mathbf{n}=\mathbf{6 1})\end{array}$ & $\begin{array}{c}\mathbf{2 0 0 0} \\
(\mathbf{n}=\mathbf{7 7})\end{array}$ & $\begin{array}{c}\mathbf{2 0 0 1} \\
(\mathbf{n}=\mathbf{8 7})\end{array}$ & $\begin{array}{c}\text { Total } \\
(\mathbf{n}=\mathbf{2 2 5})\end{array}$ \\
\hline SAS & 6.6 & 3.9 & 9.2 & 6.7 \\
Non TGA & 60.7 & 77.9 & 62.1 & 67.1 \\
Non HSD/PBS & 16.4 & 5.2 & 14.9 & 12.0 \\
PBS & 16.4 & 10.4 & 11.5 & 12.4 \\
Unknown & - & 1.3 & 2.3 & 1.3 \\
Other & - & 1.3 & - & 0.4 \\
\hline
\end{tabular}

SAS = special access scheme; $T$ TA $=$ therapeutic goods administration; $\mathrm{HSD}=$ highly specialised drugs; $\mathrm{PBS}=$ pharmaceutical benefits scheme

2000 the percentage requested was $48 \%$, however, the response rates were similar to both 1999 and 2000 .

Applications for IPU were approved by the DTC or delegated to the Chairman of the DTC or the Director of Pharmacy. The Director of Pharmacy was the person who most frequently approved IPU applications $(70.6 \%)$. The proportion of approvals by the Director of Pharmacy increased over the years. For a proportion of approvals $(11 \%)$, there was no available documentation of who approved the IPU.

In 1999 , the speciality with the greatest proportion of approvals was immunology ( $23 \%$ of the 61 approvals), followed by haematology $(10 \%)$ and nephrology $(8 \%)$. In 2000 , the trend was similar with $29 \%$ of 77 approvals being for immunology, followed by haematology (12\%) and lung transplant $(9 \%)$. As in previous years, immunology had the highest proportion in $2001(23 \%)$ followed by lung transplant $(13 \%)$ and haematology $(10 \%)$.

As the number of approvals increased, so has the cost of the IPU scheme. Between 1999 and 2001 the costs have doubled (Figure 3). As the cost has increased each year, so has the proportion of the drug expenditure being consumed by the IPU scheme. The proportion of drug expenditure on IPU increased significantly $(p<0.001)$ from $1.6 \%$ in 1999 to $3.6 \%$ in 2001 .

\section{DISCUSSION}

There was an apparent trend for the number of approvals to increase over the years reviewed. The majority of these approvals $(67.1 \%)$ were for off-iabel indications. Similar percentages have been found in other hospitals in New South Wales.'

Only $18 \%$ of the requests regarding clinical outcome of the patient were reported by clinicians. In this study there was no attempt by the investigator to separately review outcomes through medical records. This would not be feasible in routine practice, and would not reflect the purpose of the scheme.

It was noted that the pattern of drugs submitted for IPU approval have changed through the years (Table 1). Not only newly available agents were requested but also older drugs such as cyclosporin, mycophenolate and tacrolimus for varying off-label indications. As such the process of IPU review was still required to ensure optimal drug use and this may have influenced the request patterns of drugs involved.

The documentation process allowed for the cost issue of the IPU scheme to be analysed. The financial 


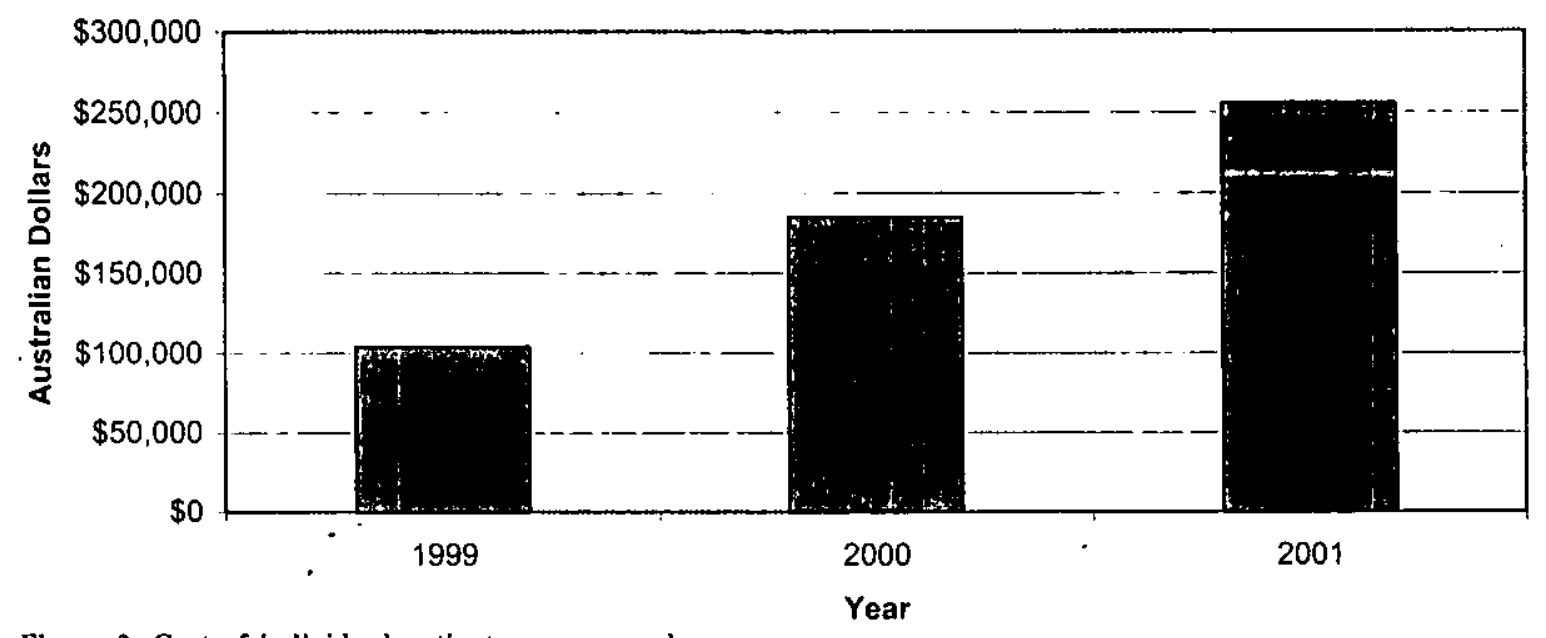

Figure 3. Cost of individual patient use approvals

impact of the IPU scheme was substantial and increased significantly $(\mathrm{p}<0.001)$ from $1.6 \%$ of the drug expenditure in 1999 to $3.6 \%$ in 2001 . As an incidental finding, the Director of Pharmacy made the majority of the approvals and there was a trend that the workload associated with this increased over the years. It must be borne in mind that the cost of the scheme not only involves the cost of the drugs but also the cost of the personnel administering this program. As a result of this review the definition of high-cost drug for the purpose of the IPU scheme was adopted as above $\$ 5000$ per patient in any given year.

It might be suggested that the overall impact of the IPU scheme, when compared with the entire drug expenditure, is minimal. However, when dealing with a capped budget and scarce resources, the question has to be asked, "Is it a fair distribution of resources to spend $3.6 \%$ of drug expenditure on only 79 patients?' The decisions are not simply based on financial cost alone, as ethical aspects play a significant role. The literature to date has focused on different mechanisms of rationing and principles such as distributive justice and human resource allocation decisions. ${ }^{11,12}$ Still, it is difficult to place value on a patient's wellbeing, and to measure the impact it has for society. It seems that in some circumstances the 'rule of rescue' is applied to high-cost drugs. This means accepting some cost-ineffective interventions for patients with rare catastrophic illness who have no other treatment options. ${ }^{13}$ Ongoing research is required to explore the decision-making process with regards to high-cost drugs.

Analysis of the current IPU scheme showed the need for recording, monitoring, and evaluating the system and other programs that influence drug costs. The results of the project indicated that the number of approvals and submissions for IPU had a tendency to increase over time. The financial impact of the scheme was significant and increased over the years studied. Revision and ongoing improvement of the current system should be considered in order to ensure efficient management of this scheme.

Guidelines could be developed, implemented and evaluated for this IPU scheme, which may then have application in other Australian hospitals.

At St Vincent's Hospital, subsequent to this review, a new policy and procedure for managing requests for high-cost drugs for IPU was established. A high-cost drugs subcommittee (operating under the auspices of the DTC) was created to act as a forum for priority setting and a way of improving the decision-making process.

\section{Acknowledgments}

We express gratitude to Maureen Heywood and Vinh Bui for undertaking this study

\section{Competing interests: None declared}

\section{References}

1. Commonwealth Department of Human Services and Health. Schedule of pharmaceutical benefits for approved pharmacists and medical practitioners. Canberra: Publications Unit; 2001.

2. Victorian Government Department of Human Services. Victoria-public hospitals and mental health services policy funding guidelines 2002-2003. Melbourne: Victorian Government Department of Human Services; 2002. Available from: <www.health.vic.gov.au/pfg2002/sectiona.pdf>.

3. Donato R, Scotton R. The Australian health care system. In: Mooney G, Scotton $R$, editors. Economics and Australian health policy. Sydney: Allen \& Unwin; 1999. p. 20-39.

4. Allinson Y. Funding of pharmaceuticals in public hospitals: PBS under the probe. Healthcover $2001 ; 11(2)$

5. Macintyre RC, Sindhusake D. Rubin G Modeling strategies for reducing pharnaceutical costs in hospital. Int J Quality in Health Care 2001; 13: 63-9. 6. Pacey $\mathrm{S}, \mathrm{Li}$ Wan Po A. A review of methods of drug cost management in hospitals. J Clin Pharm Therapeutics 1998; 23: 171-77.

7. Commonwealth Department of Health and Aged Care. Schedule of pharnaceutical benefits. Canberra: Publications Unit; 1999.

8. Commonwealth Department of Health and Aged Care. Schedule of phamaceutical benefits. Canberra: Publications Unit; 2000.

9. Commonwealth Department of Health and Aged Care. Schedule of pharmaceutical benefits. Canberra: Publications Unit; 2001.

10. Australian Medicines Handbook Pty Ltd. Australian Medicines Handbook. Adelaide: Australian Medicines Handbook Pty Lid; 2000.

11. Capp S, Savage $S$, Valeric $C$. Exploring distributive justice in health care Aust Health Rev 2001; 24: 40-44.

12. Harvery R. And on the cover of the guide were two words in red-don't panic. Aust Health Rev 2001; 24: 15-20.

13. Hill S, Mitchell AS, Henry D. Problems with interpretation of pharmacoeconomic analyses: a review of submissions to the Australian pharmaceutical benefits scheme. JAMA 2000; 283: 2116-21.

Submitted: April 2003

Accepted after external peer review: March 2004 